\title{
Interactions of Magainin-2 Amide with Membrane Lipids
}

\author{
Krzysztof Murzyn, Tomasz Róg, and Marta Pasenkiewicz-Gierula \\ Molecular Modelling Group, Faculty of Biotechnology, Jagiellonian University, ul. \\ Gronostajowa 7, Kraków, Poland \\ \{murzyn, tomekr, mpg\}@mol.uj.edu.pl
}

\begin{abstract}
Magainin-2 is a natural peptide that kills bacteria at concentrations that are harmless to animal cells. Molecular modelling methods were applied to investigate basic mechanisms of magainin-2 amide (M2a) membrane selectivity. Three computer models of a lipid matrix of either animal or bacterial membrane containing M2a were constructed and simulated. Specific interactions between membrane lipids and M2a peptides, responsible for M2a selectivity are elucidated.
\end{abstract}

\section{Introduction}

Magainin-2 (M2, GIGKFLHSAKKFGKAFVGEIMNS) is a natural, 23-amino acid cationic peptide expressed in the skin of an African frog Xenopus leavis. M2 selectively kills bacteria at concentrations that are harmless to animal cells. In organic solvents and in the vicinity of a lipid bilayer, M2 forms an $\alpha$-helix of a distinct hydrophobic moment [1]. Such a helix possesses a polar and non-polar face. At the physiological conditions, the total electrostatic charge of M2 amide (M2a), which was used in this study, is +4 e. It results from the positively charged $\mathrm{N}$-terminus, four positively charged Lys and one negatively charged Glu residues. By physical interactions with the membrane lipids, M2a disturbs the lamellar structure of the lipid matrix of biomembranes. The extent of this disturbance depends on the lipid composition of the membrane, particularly on the content of anionic lipids, as well as the peptide-tolipid ratio.

The lipid matrix of the animal plasma membrane consists mainly of neutral phosphatidylcholine (PC) and cholesterol (Chol) molecules. In contrast, the main lipid components of the bacterial membrane are neutral phosphatidylethanolamine (PE) and negatively charged phosphatidylglycerol (PG) molecules. Due to a positive charge, the effect of M2a molecules on the bacterial membrane is stronger than on the animal membrane. In the initial stage of interaction with the membrane, M2a molecules locate on the outer leaflet of the cell membrane. In the animal membrane, interactions between $\mathrm{M} 2 \mathrm{a}, \mathrm{PC}$, and Chol are such that push peptide molecules away from the surface. In the bacterial membrane, when the peptide-to-lipid (P/L) ratio is above 1:40 [2], M2a together with PG form large openings in the membrane [2, 3]. The openings (toroidal pores) consist of 4 - 7 M2a and several PG molecules. 
In this study, molecular modelling methods were applied to investigate basic mechanisms of M2a membrane selectivity. This selectivity results from specific interactions between membrane lipids and M2a peptides. Three computer models of membrane systems were constructed and simulated. The first and second systems constituted, respectively, model animal and bacterial membranes each containing two M2a molecules located horizontally on the membrane surface (carpet models, EUCARPET and PROCARPET, respectively). The third system consisted of a model bacterial membrane containing five M2a molecules that together with PG molecules formed a toroidal pore in the membrane centre (PORE). Molecular dynamics (MD) simulations of the carpet models were carried out for $12 \mathrm{~ns}$ and of PORE for $5 \mathrm{~ns}$.

\section{Methods}

\subsection{Simulation Systems}

As a model of the animal membrane, a 1-palmitoyl-2-oleoyl-phosphatidylcholine (POPC) bilayer containing 23 mol\% cholesterol (Chol) was used. Both POPC and Chol are major constituents of the animal plasma membrane. Details concerning the POPC-Chol bilayer construction are given in [4]. The EUCARPET system contained 68 POPC, twenty Chol, two M2a, eight $\mathrm{Cl}^{-}$, and 2533 water molecules (12647 atoms in total). Chlorine ions were added to neutralise +8 e charge on two M2a molecules. As a model of the bacterial membrane, a bilayer made of 1-palmitoyl-2-oleoylphosphatidylethanolamine (POPE) and 1-palmitoyl-2-oleoyl-phosphatidylglycerol (POPG) in ratio 3:1 was used. Such a lipid composition is typical for the inner bacterial membrane. The PROCARPET system contained 66 POPE, twenty two POPG, two M2a, fourteen $\mathrm{Na}^{+}$, and 2614 water molecules (12414 atoms in total). Fourteen sodium ions together with two M2a molecules neutralised -22 e charge on POPG molecules. Details concerning the POPE-POPG bilayer construction are given in [5]. The PORE system contained 138 POPE, 46 POPG (25 mol\% POPG), five M2a, twenty six $\mathrm{Na}^{+}$, and 5909 water molecules (28457 atoms in total). The toroidal pore was built according to the model proposed by Ludtke et al. [2] of five M2a and twenty POPG molecules (Fig. 1). In the pore, lipids interpose magainin helices oriented perpendicular to the membrane surface such that the polar faces of the amphiphilic helices and the polar heads of the lipids constitute the pore lining. As a result, both membrane leaflets form a continuous surface, which allows for free diffusion of lipids between the outer and inner membrane layers. Details concerning the construction of PORE are given in [6].

\subsection{Simulation Parameters}

For POPC, POPE, POPG, M2a, and Chol, optimised potentials for liquid simulations (OPLS) [7], for water, TIP3P [8], and for sodium and chlorine ions, Aqvist's parameters were used. Phospholipid, peptide, $\mathrm{Na}^{+}$, and $\mathrm{Cl}^{-}$molecules were treated as the solute molecules and water was the solvent. The united-atom approximation was applied to the $\mathrm{CH}, \mathrm{CH}_{2}$, and $\mathrm{CH}_{3}$ groups of the peptide and lipid molecules. All polar groups of the solute and solvent molecules were treated in full atomic detail. The nu- 
merical values for the atomic charges of POPE and POPG are given in [5] and of POPC follow those in Charifson et al. [9]. To retain the double bond in the oleoyl chain of phospholipids in the cis conformation, restraints on the double bond dihedral were imposed with a force constant of $100 \mathrm{kcal} \cdot \mathrm{mol}^{-1} \cdot \mathrm{rad}^{-2}$. Restraints acted whenever the deviation from an ideal conformation of $0^{\circ}$ exceeded $\pm 30^{\circ}$. Procedures for supplementing the original OPLS base with the missing parameters for the lipid headgroup were described by Pasenkiewicz-Gierula et al. [10], and those for the $\beta$-chain sp2 carbon atoms by Murzyn et al. [11]. Chiral centres in POPE, POPG, and POPC molecules were kept in a chosen configuration by defining relevant improper torsions. Improper torsions were parameterised in the OPLS forcefield with the half of the potential barrier equal to $14.0 \mathrm{kcal} \cdot \mathrm{mol}^{-1} \cdot \mathrm{rad}^{-2}$, periodicity of 3 and the energy maximum at $0^{\circ}$.

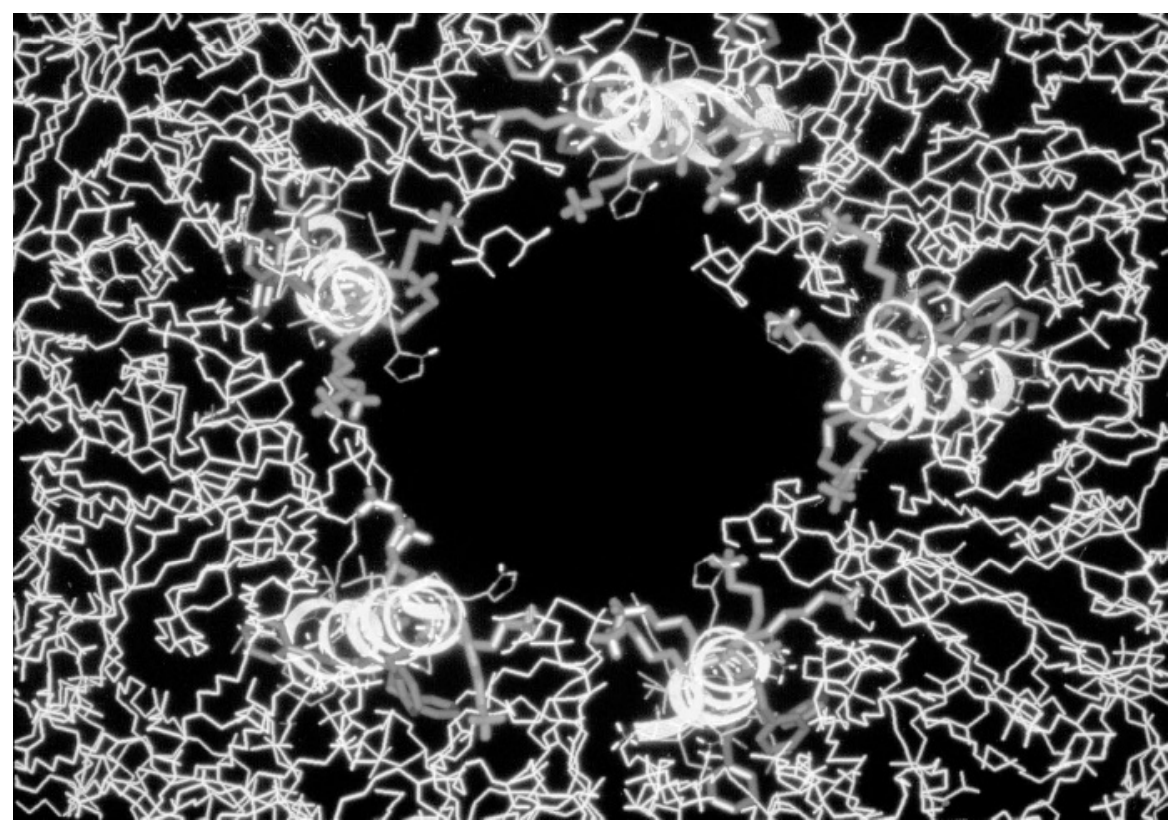

Fig. 1. Top view of M2a-POPG toroidal pore in the bacterial membrane. M2a molecules are shown as light ribbons, the Lys and Phe residues are shown as sticks, and phospholipid molecules (mainly POPG) are shown in white as lines. For clarity, water molecules were removed

\subsection{Simulation Conditions}

All three bilayer systems contain charged molecules and ions, therefore, in the MD simulations, long-range electrostatic and van der Waals interactions were evaluated by means of the Particle-Mesh-Ewald (PME) summation method [12]. A real cutoff of $12 \AA$, with a $\beta$-spline interpolation order of 5 , and a direct sum tolerance of $10^{-6}$ were used. Three-dimensional periodic boundary conditions were employed. The 
simulations were carried out using AMBER 5.0 [13]. The SHAKE algorithm [14] was used to preserve the bond lengths of the $\mathrm{OH}, \mathrm{NH}$, and $\mathrm{NH}_{2}$ groups of water and peptide molecules. The list of nonbonded pairs was updated every 25 steps.

The MD simulations were carried out at a constant pressure (1 atm) and at a temperature of $310 \mathrm{~K}\left(37^{\circ} \mathrm{C}\right)$. The temperatures of the solute and solvent were controlled independently by the Berendsen method [15]. The applied pressure was controlled anisotropically, each direction being treated independently with the trace of the pressure tensor kept constant for $1 \mathrm{~atm}$, again using the Berendsen method [15]. The relaxation times for temperatures and pressure were set at 0.4 and $0.6 \mathrm{ps}$, respectively. The bilayers were simulated for $12 \mathrm{~ns}$ (EUCARPET and PROCARPET) and $5 \mathrm{~ns}$ (PORE). For analyses, last 10-ns fragments of EUCARPET and PROCARPET trajectories and the last 3-ns fragment of PORE trajectory were used. The average values given below are ensemble and time averages obtained from the block averaging procedure. Errors in the derived average values are standard error estimates.

\subsection{Analyses}

The instantaneous orientation of the peptide molecules in the bilayers was monitored using a rigid body quaternion fit as implemented in MMTK package [16]. Details are given in [6]. The reference helical structure of the M2a molecule was the experimentally determined peptide structure [1].

\section{Results}

\subsection{Short Distance Lipid-Peptide Interactions}

\subsubsection{Animal Membrane}

In EUCARPET, M2a molecules are positioned in the interfacial region of the membrane. Such localisation allows them to interact both with the polar and non-polar fragments of POPC and Chol molecules. The side-chains of polar and charged amino acids interact with the phosphate and carbonyl groups of POPC and the hydroxyl group of Chol via direct hydrogen bonds (H-bond) and water bridges (WB). The largest contribution to M2a-POPC polar interactions have two Lys residues (Lys10 and 14), and to M2a-Chol polar interactions has Glu19 (Fig. 2). The side-chains of nonpolar residues and non-polar fragments of POPC and Chol interact via van der Waals interactions. The largest contribution to M2a-POPC non-polar interactions have mainly aromatic residues (Phe7, 12, and 16), and to M2a-Chol non-polar interactions mainly aliphatic residues (Ile2, Leu6, and Val17). Numbers of M2a-lipid interactions are given in Table 1.

\subsubsection{Bacterial Membrane}

In PROCARPET, localisation of M2a molecules is similar to that in EUCARPET. Nevertheless, non-polar interactions between M2a and phospholipids are significantly stronger than in EUCARPET, particularly those of Phe12 and Phe16. The number of direct M2a-lipid H-bonds is similar in both membranes, whereas the number of M2a- 
lipid water bridges is much less than in EUCARPET. The largest contribution to M2a-lipid polar interactions have Lys4, 10, and 11, and Gly19. Numbers of M2a-lipid interactions are given in Table 1.

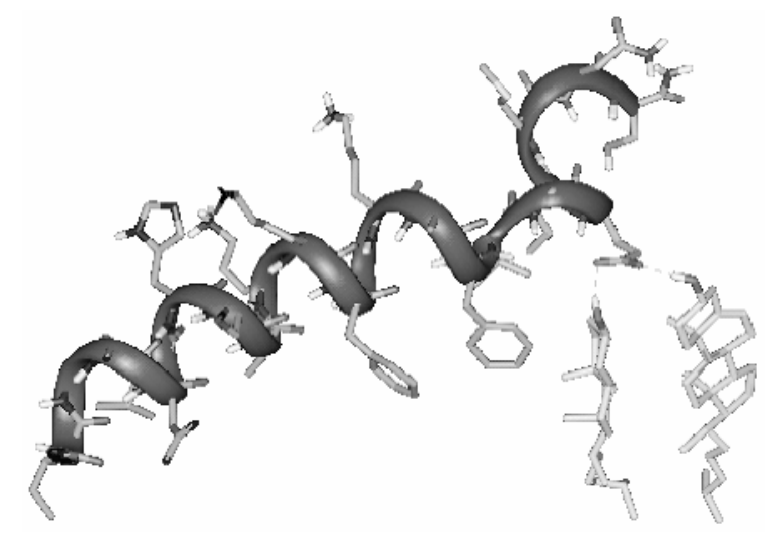

Fig. 2. One of two M2a molecules in the animal membrane together with two Chol molecules that are simultaneously H-bonded to Glu19. M2a is shown as dark ribbon, the residues and Chol molecules are shown as sticks

Table 1. Average numbers of hydrogen bonds (H-bonds) and water bridges (WB) between a M2a and surrounding lipids, as well as carbon atoms in phospholipid or Chol molecules that are within $7.5 \AA$ from any atom of $\mathrm{M} 2 \mathrm{a}$ non-polar residues (non-polar interactions, NP) in EUCARPET, PROCARPET and PORE. Errors are standard error estimates

\begin{tabular}{lcclc}
\hline & \multicolumn{2}{c}{ EUCARPET } & \multirow{2}{*}{ PROCARPET } & PORE \\
\cline { 2 - 3 } & POPC & CHOL & & \\
\hline H-bonds & $6.3 \pm 0.4$ & $1.1 \pm 0.1$ & $7.0 \pm 0.5$ & $7.2 \pm 0.6$ \\
\hline WB & $11.4 \pm 0.8$ & $1.8 \pm 0.2$ & $7.1 \pm 0.4$ & $7.9 \pm 0.5$ \\
\hline NP & $1138 \pm 45$ & $93 \pm 4$ & $1301 \pm 50$ & $1155 \pm 40$ \\
\hline
\end{tabular}

\subsubsection{Toroidal Pore}

In PORE, M2a molecules together with POPG headgroups form a toroidal pore. Even though M2a are located perpendicular to the bilayer surface, their local environment is similar to the interfacial region of PROCARPET. Consequently, the pattern of polar and non-polar interactions between peptide and lipid molecules are to a large extent similar to those in PROCARPET. Numbers of M2a-lipid interactions are given in Table 1. The strongest contribution to polar interactions arises from all four Lys residues, whereas to non-polar interactions arises form all three Phe residues. 


\subsection{Orientation and Conformation of Peptide Molecules in the Membrane}

\subsubsection{Animal Membrane}

The M2a molecules in EUCARPET remain nearly parallel to the bilayer surface and their orientation is similar to the initial one. Strong H-bonding between two Chol molecules and Glu19 of one M2a molecule (Fig. 2) results in a local loss of helicity of this molecule.

\subsubsection{Bacterial Membrane}

The M2a molecules in PROCARPET also remain nearly parallel to the bilayer surface nevertheless, their orientation differs from the initial one. The peptides rotated about their long axes by an average angle of $\sim 15^{\circ}$.

\subsubsection{Toroidal Pore}

The M2a molecules in PORE fluctuate about the orientation perpendicular to the membrane surface. Like in PROCARPET, the peptide molecules underwent limited rotation about their long axes.

\section{Conclusions}

1. Interactions between Chol and M2a in EUCARPET impeded favourable interaction of the peptide with membrane phospholipids. On the longer time scale, this may lead to desorption of the peptide from the membrane surface.

2. M2a molecules in PROCARPET, by rotating about their long axes, assumed orientation that maximises their interaction with both polar and non-polar groups of phospholipids. These interactions affect the organisation of both interfacial and hydrophobic membrane region. On the longer time scale, this may lead to local destruction of the membrane lamellar structure.

3. M2a-lipd interactions in PORE are similar to those in PROCARPET. These interactions stabilize the pore structure.

Acknowledgements. This work was supported by grants 6 P04A 04116 , 6 P04A 03121 and KBN/SGI ORIGIN 2000/UJ/048/1999 from the Committee for Scientific Research and partially by European Union (contract no. BIER ICA1-CT2000-70012). KM and TR acknowledge a fellowship award from the Polish Foundation for Science.

\section{References}

1. Gesell, J., Zasloff, M., Opella, S.J.: Two-dimensional H-1 NMR experiments show that the 23-residue magainin antibiotic peptide is an alpha-helix in dodecylphosphocholine micelles, sodium dodecylsulfate micelles, and trifluoroethanol/water solution. J. Biomol. NMR. 9 (1997) 127-135 
2. Ludtke, S.J., He, K., Wu, Y., Huang, H.: Cooperative membrane insertion of magainin correlated with its cytolytic activity. Biochim. Biophys. Acta 1190 (1994) 181-184

3. Matsuzaki, K., Murase, O., Furii, N., Miyajima, M.: An antimicrobial peptide, magainin 2, induced rapid flip-flop of phospholipids coupled with pore formation and peptide translocation. Biochemistry 35 (1996) 11361-11368

4. Róg, T.: Effects of cholesterol on the structure and dynamics of phospholipid bilayers: a molecular dynamics simulation studies. Ph.D. thesis. Jagiellonian University, Poland (2000) 1-147

5. Murzyn, K., Pasenkiewicz-Gierula, M.: Construction and optimisation of a computer model for a bacterial membrane. Acta Biochim. Pol. 46 (1999) 631-639

6. Murzyn, K., Pasenkiewicz-Gierula, M.: Construction of a toroidal model for the magainin pore. J. Mol. Mod. 9 (2003) 217-224

7. Jorgensen, W.L., Tirado-Rives, J.: The OPLS potential functions for proteins. Energy minimizations for crystals of cyclic peptides and crambin. J. Am. Chem. Soc. 110 (1988) 1657-1666

8. Jorgensen, W.L., Chandrasekhar, J., Madura, J.D., Impey, R.W., Klein M.L.: Comparison of simple potential functions for simulating liquid water. J. Chem. Phys. 79 (1983) 926-935

9. Charifson, P.S., Hiskey, R.G., Pedersen, L.G.: Construction and molecular modeling of phospholipid surfaces. J. Comp. Chem. 11 (1990) 1181-1186

10. Pasenkiewicz-Gierula, M., Takaoka, Y., Miyagawa, H., Kitamura, K., Kusymi, A.: Charge pairing of headgroups in phosphatidylcholine membranes: A molecular dynamics simulation study. Biophys. J. 76 (1999) 1228-1240

11. Murzyn, K., Róg, T., Jezierski, G., Takaoka, Y., Pasenkiewicz-Gierula, M.: Effects of phospholipid unsaturation on the membrane/water interface: a molecular simulation study. Biophys. J. 81 (2001) 170-183

12. Essmann, U., Perera, L., Berkowitz, M.L., Darden, T., Lee, H., Pedersen, L.G.: A smooth particle mesh Ewald method. J. Chem. Phys. 103 (1995) 8577-93.

13. Case, D.A., Pearlman, D.A., Caldwell, J.W., Cheatham III, T.E., Ross, W.S., Simmerling, C., Darden, T.A., Merz, K.M., Stanton, R.V., Cheng, A.L., Vincent, J.J., Crowley, M., Ferguson, D.M., Radmer, R.J., Seibel, G.L., Singh, U.C., Weiner, P.K., Kollman, P.A.: AMBER 5.0. University of California, San Francisco (1997)

14. Ryckaert, J. P., Cicotti, G., Berendsen, H.J.C.: Numerical integration of the Cartesian equations of motion of a system with constraints: molecular dynamics of n-alkanes. J. Comp. Phys. 22 (1977) 327-341

15. Berendsen, H.J.C., Postma, J.P.M., van Gunsteren, W.F., DiNola, A., Haak, J.R.: Molecular dynamics with coupling to an external bath. J. Chem. Phys. 81 (1984) 3684-3690

16. Hinsen, K.: The molecular modeling toolkit: A new approach to molecular simulations. J. Comp. Chem. 21 (2000) 79-85 